\title{
NONLINEAR MAPS PRESERVING HIGHER-DIMENSIONAL NUMERICAL RANGE OF SKEW LIE PRODUCT OF OPERATORS
}

\author{
Chaoqun Chen and Fangyan Lu
}

Abstract. Let $k$ be a positive integer. Let $H$ and $K$ be complex Hilbert spaces of dimensions greater than $k$. By $W_{k}(A)$ denote the $k$-dimensional numerical range of an operator $A$. In this paper we prove that a surjective map $\phi: B(H) \rightarrow B(K)$ satisfies $W_{k}\left(A B-B A^{*}\right)=$ $W_{k}\left(\phi(A) \phi(B)-\phi(B) \phi(A)^{*}\right)$ for all $A, B \in B(H)$ if and only if there exists a unitary operator $U \in B(H, K)$ such that $\phi(A)=\gamma U A U^{*}$ for all $A \in B(H)$, where $\gamma \in\{-1,1\}$.

Mathematics subject classification (2010): 47B48, 46L10.

Keywords and phrases: Higher-dimensional numerical range, skew Lie product.

\section{REFERENCES}

[1] C. Akemann And J. Anderson, The spectral scale and the numerical range, Internat. J. Math. 14 (2003), 171-189.

[2] C. Akemann And J. Anderson, The spectral scale and the $k$-numerical range, Glasg. Math. J. 45 (2003), 225-238.

[3] M. BReŠAR AND M. Fošner, On rings with involution equipped with some new product, Publ. Math. Debrecen. 57 (2000), 121-134.

[4] M. A. Chebotar, Y. Fong And P. H. LeE, On maps preserving zeros of the polynomial $x y-y x^{*}$, Linear Algebra Appl. 408 (2005), 230-243.

[5] J. L. CUI AND J. C. Hou, Linear maps preserving elements annihilated by a polynomial $X Y-Y X^{\dagger}$, Studia Math. 174 (2006), 183-199.

[6] J. L. CUI AND C. K. LI, Maps preserving product $X Y-Y X^{*}$ on factor von Neumann algebras, Linear Algebra Appl. 431 (2009), 833-842.

[7] J. L. CUI, Q. Li, J. Hou AND X. QI, Some unitary similarity invariant sets preserves of skew Lie products, Linear Algebra Appl. 457 (2014), 76-92.

[8] J. L. CUI AND C. PARK, Maps preserving strong skew Lie product on factor von Neumann algebras, Acta Math. Sci. 32 (2012), 531-538.

[9] P. A. Fillmore AND J. P. William, Some convexity theorems for matrices, Glasgow Math. J. 12 (1971), 110-117.

[10] H. GAU AND C. K. LI, $C^{*}$-isomorphisms, Jordan isomorphisms, and numerical range preserving maps, Proc. Amer. Math. Soc. 135 (2007), 2907-2914.

[11] M. GOLDBERG AND E. STRAUS, Inclusion relations involving $k$-numerical ranges, Linear Algebra Appl. 15 (1976), 261-270.

[12] K. GUSTAFSON AND D. RAO, Numerical ranges: The field of values of linear operators and matrices, Springer, New York, 1997.

[13] P. R. Halmos, A Hilbert Space Problem Book, 2nd ed., Springer Verlag, New York. 1982.

[14] C. K. LI, Linear operators preserving the higher numerical radius of matrices, Linear and Multilinear Algebra. 21 (1987), 63-73.

[15] C. K. LI, A survey on linear preservers of numerical ranges and radii, Taiwanese J. Math. 5 (2001), 477-496.

[16] F. LU, Linear dependence of operators characterized by trace functionals, Linear Algebra Appl. 434 (2011), 343-355. 
[17] M. Marcus, N. Moyls And I. FilipPenko, Normality and the higher numerical range, Canad. J. Math. 30 (1978), 419-430.

[18] L. MolnÁR, A condition for a subspace of $B(H)$ to be an ideal, Linear Algebra Appl. 235 (1996), 229-234.

[19] L. MolnÁR AND P. ŠEMRL, Nonlinear commutativity preserving maps on self-adjoint operators, Quart. J. Math. 56 (2005), 589-595.

[20] M. OMLADIČ, On operators preserving the numerical range, Linear Algebra Appl. 134 (1990), 3151.

[21] S. PieRCE AND W. WATKIns, Invariants of linear maps on matrix algebras, Linear and Multilinear Algebra. 6 (1978), 185-200.

[22] X. QI AND J. HOU, Strong skew commutativity preserving maps on von Neumann algebras, J. Math. Anal. Appl. 397 (2013), 362-370.

[23] P. ŠEMrL, On Jordan*-derivations and an application, Colloq. Math. 59 (1990), 241-251.

[24] P. ŠEMRL, Quadratic functionals and Jordan*-derivations, Studia Math. 97 (1991), 157-165. 